\title{
Dispositif d'enregistrement en continu du comportement alimentaire journalier et étude de la variabilité du comportement chez la vache laitière *
}

\author{
$V$ Girard ${ }^{\star *}, J$ Labonté \\ Ministère de l'Agriculture, des Pêcheries et de l'Alimentation, \\ direction de la recherche et du développement, Service de la zootechnie, \\ 120-A chemin du Roy, Deschambault, Québec, GOA 150Canada
}

(Reçu le 12 février 1992; accepté le 1er juillet 1992)

Résumé - La vitesse d'ingestion d'aliments (ING, $\mathrm{kg} \mathrm{min}^{-1}$ ) et la fréquence de mastication pendant la rumination (CMR, coups de mâchoire $\mathrm{min}^{-1}$ ) de 4 vaches, recevant une fois par jour (14 h) une ration à base d'ensilage de fléole, ont été enregistrées en continu. Cette technique mesure les coups de mâchoire à l'aide d'un signal numérique généré par un interrupteur magnétique installé sur le licol. La distinction entre les périodes de rumination et celles d'ingestion est basée sur l'état du poids des auges : lorsque le poids diminue, il ne peut y avoir rumination. Les poids enregistrés alors que la tête est dans l'auge sont ignorés. L'algorithme de traitement des données compte CMR et ING, avec un coefficient de variation de $2,9 \%$ et $2,2 \%$ respectivement. Cette technique a été utilisée pour mesurer la variabilité du comportement alimentaire pendant deux périodes de 9 jours à 1 mois d'intervalle. À partir du premier repas qui suit la distribution d'aliments, les CMR et les ING ont été totalisés pendant $24 \mathrm{~h}$. Chaque profil comprenait 12 observations régulièrement réparties de $1,5 \mathrm{~h}$ à 24 h. Pour chacune des observations, on a calculé une moyenne de 3 ou de 2 j consécutifs et obtenu ainsi 3 ou 4 répétitions des profils journaliers par période. L'effet du nombre de répétition par période sur la variabilité des profils journaliers a été obtenu en analysant les profils à l'aide d'un dispositif en "split-plot". Le nombre de jours par répétition n'a pas modifié le coefficient de variation individuelle du profil d'ingestion $(4 \%)$, ni celui de rumination $(9,1 \%)$. La variabilité peu importante des profils démontre la constance des différences individuelles pendant des périodes de $9 \mathrm{j}$.

vache laitière / ingestion / mastication / enregistrement automatique / comportement alimentaire / vache laitière

Summary - Continuous registering device for daily feeding behavior and study of behavior variability in the dairy cow. Rate of intake (ING, $\mathrm{kg} \mathrm{mir}^{-1}$ ) and frequency of jaw movements for rumination (CMR, movements min $^{-1}$ ) of 4 cows in late lactation stall-fed once daily (14 h) with timothy silage were continuously registered. Jaw movements were counted with a magnetic switch attached

\footnotetext{
- Publication 381, Direction de la recherche et de l'enseignement, ministère de l'Agriculture, des Pêcheries et de l'Alimentation.

"* Correspondance et tirés à part : service de la zootechnie, 120-A, chemin du Roy, Deschambault, Québec, GOA 1SO, Canada.
} 
to the halter. Valid manger weights were recorded when the cow raised its head from the manger. ING was computed from manger weight reduction. By simultaneously monitoring manger weights and jaw movement frequencies, chewing was attributed either to CMR or intake. The resulting coefficients of variation were $2.2 \%$ for ING and $2.9 \%$ for CMR. This technic was then used to estimate the variability of individual feeding and ruminating behaviors during two 9-d periods at a 1-month interval. Starting with the first meal, the amount of CMR and ING was assessed for $24 \mathrm{~h}$. Individual patterns were established on the basis of 12 observations regularly spaced between 1.5 and $24 \mathrm{~h}$. These 12 observations were individually averaged over 2 or $3 d$ repetitions. Thus periods were split either into 3 or 4 repetitions. Behavior variability was estimated by analyzing the pattern with a split-plot design. The length of the repetition did not modify the variation coefficient of intake $(3.9 \%)$ and rumination behavior (8.7\%). Detection of different behaviors was possible since individual patterns persisted over 9-d periods with little variation.

dairy cow / intake rate / jaw movement / automatic registration / feeding behaviour / dairy cow

\section{INTRODUCTION}

Les activités alimentaires de la vache laitière entre 2 distributions d'aliment se composent généralement d'un repas principal et de plusieurs petits repas secondaires entrecoupés par des périodes d'activités méryciques ou de repos masticatoire. L'importance du repas principal, qui peut représenter jusqu'à $80 \%$ de l'ingestion volontaire, et la vitesse d'ingestion (Baumont et al, 1989) sont modifiées par la qualité du fourrage (Baumont et al, 1989) et sont reliées à l'ingestion volontaire (Deswysen, 1987). La fréquence et la durée des repas et des périodes de rumination modifient la distribution et la réduction de la taille des particules alimentaires nécessaires à leur digestion et à leur transit (Deswysen, 1987; Martz et Belyea, 1986). L'enregistrement en continu des repas et des périodes de rumination permet d'établir le profil du comportement alimentaire.

La variation intra-individuelle du comportement alimentaire, c'est-à-dire la variation individuelle d'un jour à l'autre, est toujours plus faible que la variation entre deux individus (Vasilatos and Wangsness, 1980; Dulphy et al, 1990; Deswysen, 1987), laquelle est peut-être d'origine génétique (Hancock, 1953; Deswysen, 1987). Par conséquent, on devrait pouvoir démontrer que les mesures du comportement alimentaire individuel peuvent être répétées.

On peut distinguer les coups de mâchoire des périodes d'ingestion et ceux des périodes de rumination par l'analyse de la fréquence (Luginbuhl et al, 1987) ou de la durée à une fréquence constante (Beauchemin et al, 1989; Däberitz et al, 1989). En utilisant un signal analogique proportionnel à l'ouverture des mâchoires, Beauchemin et al (1989) ont ramené l'erreur sur la durée de rumination à $1,3 \%$ seulement. Mais le signal analogique ne permet pas de compter les coups de mâchoire individuellement. II est donc nécessaire de le transformer en signal numérique à l'aide d'un critère d'ouverture minimale de la mâchoire (Beauchemin et al, 1989). L'utilisation d'un interrupteur est plus simple, mais la distinction entre les diverses activités devient difficile (Luginbuhl et al, 1987). En utilisant conjointement un interrupteur et un capteur de pesage à jauge de contrainte, nous pouvons identifier les périodes de rumination sans recourir à une programmation complexe.

Les objectifs de cette présentation sont de décrire le dispositif utilisé pour faire la distinction entre les activités d'ingestion et de rumination et de mesurer la variabilité du comportement alimentaire individuel. 


\section{MATÉRIEL ET MÉTHODES}

Quatre vaches (poids moyen $568 \pm 17 \mathrm{~kg}$, production laitière : $23,4 \pm 3,3 \mathrm{~kg}$ ), ont été choisies au hasard dans un troupeau de 24 vaches et placées dans des stalles individuelles équipées d'auge sur capteur de charge. Elles recevaient à $14 \mathrm{~h}$ une ration à base d'ensilage de fléole récoltée en fin d'épiaison et dont la teneur en matière sèche (MS) était de $35 \%$ et comprenant $25 \%$ (base de MS) d'orge et de tourteau de soja (16\% de protéine brute dans la ration mélangée). Les quantités étaient ajustées chaque jour pour permettre $10 \%$ de refus. L'eau était disponible en permanence.

\section{Description du système}

Le système d'acquisition, de traitement et d'interprétation des signaux représenté sur la figure 1 permet d'enregistrer en continu et sur une base individuelle, les coups de mâchoire et le poids des auges en stabulation entravée. Un transformateur ( $A$, fig 1) met les différents capteurs (B, C, D) sous tension. Un ordinateur scrute l'état des capteurs selon une fréquence déterminée par l'expérimentateur à l'aide de cartes d'acquisition ( $E, F, G, H$ ). Un logiciel intègre ces informations pour déterminer les coups de mâchoire pendant l'ingestion d'aliments (ING, $\mathrm{kg} \mathrm{min}^{-1}$ ) et la rumination (CMR, coups min $^{-1}$ ).

Une auge de 120 litres repose sur un capteur de pesage à jauge de contrainte ${ }^{1}$ d'une capacité de $200 \mathrm{~kg}$ (D). Les déplacements latéraux sont minimisés par une structure tubulaire (K). La vache peut exercer une pression sur le capteur de pesage mais elle doit pour cela passer la tête sous une barre de protection (J). Dans cette position, la tête interrompt le faisceau lumineux d'une celluile photoélectrique ${ }^{2}$ (C). Ce dispositif permet d'éliminer les lectures de poids lorsque l'animal a la tête dans la mangeoire.

Le compteur de coup de mâchoire (B) est composé d'un licol, d'un interrupteur ${ }^{3}$, d'une

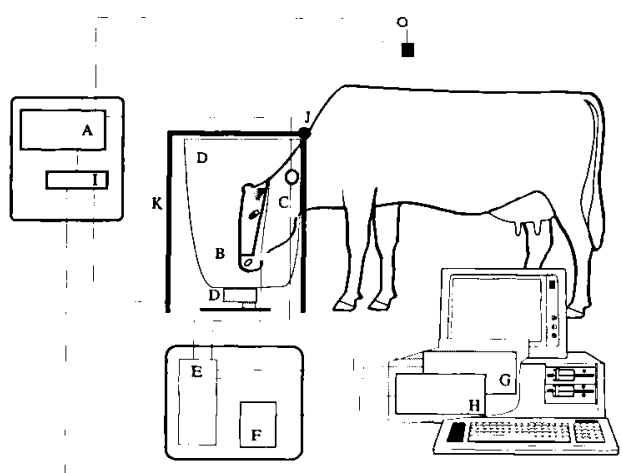

Fig 1. Système d'acquisition, traitement et interprétation des signaux pour le comptage des coups de mâchoires et l'ingestion d'aliments en continu. La vache mange dans une auge reposant sur un capteur de pesage à jauge de contrainte (D). Les signaux générés par les capteurs $(B, C, D)$ sont conditionnés (I) ou transmis (E) à des cartes d'acquisition $(H, G)$. Une carte d'expansion (F) permet d'avoir jusqu'à 8 balances.

poulie et d'un contrepoids. L'interrupteur est taillé dans un bloc de plexiglas de $6 \times 3 \times 1 \mathrm{~cm}$. Au centre et sur la longueur du bloc est percé un trou de $0,5 \mathrm{~cm}$ de diamètre dans lequel coulisse un aimant qui active un contact magnétique ${ }^{4}$. L'aimant maintenu en face du contact par un ressort est relié à une bande élastique qui passe sous la mâchoire. L'ouverture de la mâchoire exerce une pression sur la bande élastique, contracte le ressort et déplace l'aimant. Un déplacement de $0,5 \mathrm{~cm}$ et une fermeture complète des mâchoires sont nécessaires pour ouvrir et fermer le contact magnétique. Une diode lumineuse permet de vérifier le fonctionnement de l'interrupteur lorsqu'on ajuste le licol.

Le transformateur (A) envoie un courant de $10 \mathrm{~V}$ et $100 \mathrm{~mA}$ au conditionneur de signal 5 (I) qui alimente le capteur de pesage. La variation de résistance du capteur de pesage est conditionnée en un signal analogique de 0 à $20 \mathrm{~mA}$,

\footnotetext{
1 Hi-capacity platform load cell, modèle 6762, Transducers Inc, Cerritos, CA-90701, Etats-Unis.

2 Threaded barrel photoelectric controls, modèle cr17db, General Electric.

3 Mecaneuf, 202, rue Hellène, Portneuf GOA 1YO, QUE, Canada.

4 Interrupteur magnétique Reed, modèle Mini-2S-115, Newark, Laval, H7T 1S9, QUE, Canada.

${ }^{5}$ Conditionneur de signal, modèle OM3-S-3, Omega, Stamford, CT 06707-0047, Etats-Unis.
} 
qui est reçu sans perte de précision par la carte d'expansion ${ }^{1}(F)$ située à plus de $50 \mathrm{~m}$ de la balance. Une carte d'expansion peut recevoir les signaux analogiques provenant de 8 balances.

Les signaux numériques, c'est-à-dire 0 ou 12 $U$ par la cellule photo-électrique (C) et 0 ou $5 \mathrm{U}$ pour linterrupteur de licol, sont transmis à des coupleurs optiques ${ }^{2}$ (E) qui isolent l'ordinateur du circuit électrique.

\section{Acquisition des données}

Les données provenant des capteurs aboutissent sur les cartes d'acquisition qui sont insérées dans les fentes d'expansion d'un compatible IBM 80286 à $12 \mathrm{MHz}$, équipé d'un disque dur de $40 \mathrm{MB}$. La première de ces cartes est une carte d'acquisition pour données analogiques ${ }^{3}(H)$. La seconde carte est une carte d'acquisition de données numériques ${ }^{4}(G)$. Un ensemble de procédures 5 écrites en Pascal version $5^{6}$ permet de lire l'état de tous les capteurs, c'est-à-dire jusqu'à 8 vaches.

Le programme d'acquisition écrit peut travailler en arrière-plan pour afficher sur l'écran, à l'aide d'un menu, soit les résultats, soit la représentation graphique des résultats, soit l'affichage de l'état des capteurs. Afin d'améliorer les performances graphiques, la fréquence de lecture des capteurs a été limitée à $19 \mathrm{~Hz}$. En théorie, une fréquence de $4 \mathrm{~Hz}$ serait suffisante pour compter les changements d'état des interrupteurs de licol aussi longtemps que la fréquence des coups de mâchoires est inférieure à 2 coups/s. Le programme d'acquisition additionne individuellement les changements d'état de chaque interrupteur de licol pendant une minute, puis inscrit sur le disque dur le numéro de l'enregistrement et, pour chaque vache, un numéro d'identification, la fréquence des coups de mâ- choires pendant la demière minute, le poids de l'auge et l'état de la cellule photo-électrique à la fin de la minute.

\section{Traitement des données}

Le traitement des données permet de totaliser sur une base journalière l'ingestion d'aliment $\mathrm{min}^{-1}$ (ING, $\mathrm{kg} \mathrm{min}^{-1}$ ) et les coups de mâchoire pendant la rumination (CMR, coups $\min ^{-1}$ ). La somme des ING pendant $24 \mathrm{~h}$ représente l'ingestion volontaire d'ensilage.

Comme l'ingestion et la rumination s'excluent mutuellement, la rumination ne peut avoir lieu que lorsque le poids de la balance est stationnaire, c'est-à-dire pendant les périodes où les variations de poids min $^{-1}$ proviennent d'erreurs de pesée ou de pressions temporaires sur l'auge. L'erreur de pesée représente une variation aléatoire de la lecture du poids (fig 2).

Pendant les périodes de poids stationnaire, la fréquence des coups de mâchoires permet de distinguer les CMR des autres activités, comme la consommation d'eau. Dans cette expérience, on a utilisé une fréquence minimale de 35 coups de mâchoire min $^{-1}$ pour les CMR. Les temps de rumination ne sont pas surestimés par des séquences prolongées de léchage, qui ont une fréquence supérieure à 35 coups min-1 $^{-1}$, car, lorsque la langue n'est pas rentrée, la mâchoire inférieure ne se relève pas suffisamment pour activer le capteur des coups de mâchoire.

Pendant les repas, l'indicateur de position de la tête permet d'invalider les enregistrements du poids effectués lorsque la tête de la vache est dans l'auge. ING est la pente obtenue en divisant la différence de poids entre 2 pesées valides par la différence de temps écoulé entre ces 2 pesées.

\footnotetext{
1 Expansion analogique universelle, modèle exp-16, MetraByte Corporation, Taunton, MA 02194, Etats-Unis.

2 Carte opto-coupleur, modèle PB16A, Omega, Stamford, CT 06707-0047, Etats-Unis.

${ }^{3}$ Eight chanel analog input board, modèle DAS-8PGA, MetraByte Corporation, Taunton, MA 02780, Etats-Unis.

424 bit high output current parallel digital interface, modèle PIO-24, MetraByte, Taunton, MA 02780, Etats-Unis.

5 Science \& Engineering Tools, modèle IPC-TP-016, Quinn-Curtis, Needham, MA 02194.

${ }^{6}$ Borland, Scotts Valley, CA 95067-0001, Etats-Unis.
} 


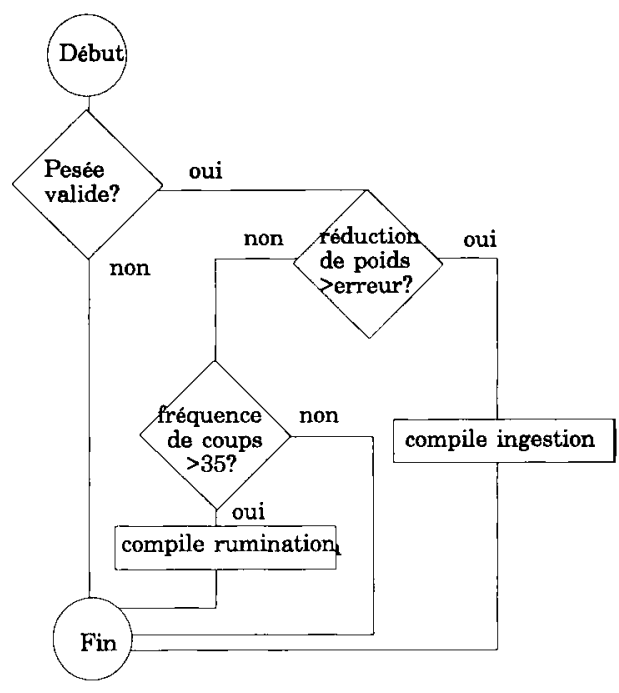

Fig 2. Algorithme de traitement des données pour la compilation des coups de mâchoire pendant la rumination et la compilation de l'ingestion.

\section{Validation de l'algorithme}

Après une période d'adaptation de $10 \mathrm{j}$, on a interrompu la rumination journellement entre $9 \mathrm{~h}$ 30 et $12 \mathrm{~h}$ pour obtenir chaque matin 3 périodes de rumination d'une durée contrôlée de 3,6 et 9 min, séparées d' 1 min ou plus d'inactivité. L'observateur interromptait la rumination en s'approchant rapidement de la tête de la vache, manipulait la tête pendant $1 \mathrm{~min}$, s'éloignait, et attendait la reprise de la rumination. Ces périodes consécutives de rumination ont été identifiées parmi les enregistrements de l'ordinateur, et les CMR ont été additionnés séparément pour chaque période. Ces CMR ont été comparés au comptage visuel de chaque période. On a aussi comparé l'ingestion volontaire obtenue par différence entre les quantités distribuées et les refus mesurés sur une balance mécanique et la somme des ING pendant $24 \mathrm{~h}$.

L'erreur des prises de données a été ensuite établie à l'aide du modèle :

$$
Y=b 0 X \pm \varepsilon
$$

avec $Y$ : mesure visuelle (ingestion volontaire ou coups de mâchoire de rumination); $X$ : mesure correspondante par l'ordinateur; $\varepsilon$ : erreur résiduelle.

L'hypothèse sur le coefficient de régression, $b^{0}=1$, a été vérifiée avec un test de Fisher (SAS, 1987).

\section{Mesure du comportement alimentaire}

Afin de décrire le comportement alimentaire, on a cumulé journellement les ING, CMR, le temps pour ING et celui pour CMR pendant 2 périodes de $9 \mathrm{j}$, à 1 mois d'intervalle. Les moyennes individuelles par période de l'ingestion volontaire des CMR cumulés et de la durée de la rumination pendant $24 \mathrm{~h}$ ont été ensuite soumises à une analyse de variance avec les vaches comme facteur. L'ingestion volontaire, le cumul des CMR et la durée de la rumination ont été ensuite corrélés.

\section{Mesure de la variabilité du comportement alimentaire}

Chaque période a été ensuite divisée en répétitions dans le temps. Les profils journaliers de ING et de CMR ont été obtenus en totalisant CMR et ING à partir du début du premier repas pendant $1,5 \mathrm{~h}$ puis toutes les $2 \mathrm{~h}$ jusqu'à la distribution d'aliment suivante $(23,5-24 \mathrm{~h})$, soit 12 observations par profil journalier. Pour chacune des obsenvations du profil journalier, on a calculé une moyenne par répétition $\left(X_{i}\right)$ représentant la moyenne des totaux de CMR ou ING après $i$ h. $X_{i j k \mid}$ représente $X_{i}$ à la $i^{\theta}$ observation du profil (HEURE $\left.E_{i}\right)$ de la je vaches $\left(V_{i}\right)$, pendant la $k^{e}$ période $\left(P_{k}\right)$ et la $P$ répétition (REPe). L'analyse statistique de $X_{i j k l}$ a été faite en assimilant les répétitions et les 12 observations du profil aux parcelles et aux sous-parcelles d'un dispositif en split-plot à l'aide de la procédure GLM de SAS (1987) :

$$
\begin{aligned}
& X_{i j k l}=V_{j}+P_{k} \pm \varepsilon_{a}+R E P_{1}+R E P_{1} V_{j}+R E P_{1}^{*} P_{k} \\
& \pm \varepsilon_{b}+\text { HEURE }_{i}+\text { HEURE }_{i}{ }^{*} V_{j}+\text { HEURE }_{i}{ }^{*} P_{k} \\
& \pm \varepsilon_{\mathrm{c}}+\mathrm{REP}_{i}{ }^{*} \mathrm{HEURE}_{\mathrm{i}}+\mathrm{REP}_{j}{ }^{*} \mathrm{HEURE}_{i}{ }^{*} V_{j} \\
& + \text { REP, }{ }^{*} \text { HEURE }{ }_{i}^{*} P_{k} \pm \varepsilon_{d}
\end{aligned}
$$


Dans ce modèle, la variation résiduelle est répartie en 4 types d'erreur, $\varepsilon_{a}, \varepsilon_{b}, \varepsilon_{c}$ et $\varepsilon_{d}$. Comme tous les $X_{i j k l}$ sont égaux à 0 en début de profil, les interactions HEURE ${ }_{i}{ }^{*} V_{i}$ (33 degrés de liberté, dl) et HEURE ${ }_{i}^{*} P_{k}(11 d)$ testées avec l'erreur $\varepsilon_{\mathrm{c}}$ (33 di) permettent d'estimer l'effet des vaches et des périodes par rapport au comportement alimentaire moyen.

Pour déterminer la variabilité du comportement alimentaire entre 2 distributions d'aliment, le coefficient de variation (CV) du profil individuel a été estimé à l'aide du carré moyen de l'interaction REP ${ }^{*}{ }^{*} H E U R E_{i}{ }^{*} V_{j}$ et de la moyenne des $\mathrm{x}_{\mathrm{ijkl} \mathrm{k}}$ :

$$
C V=100 * \frac{\sqrt{R E P_{1}{ }^{*} H E U R E_{i}{ }^{*} V_{j}}}{\overline{X_{i j k l}}}
$$

Dans cette expérience, nous avons testé l'effet des vaches à l'aide de $\varepsilon_{\mathrm{c}}$ et comparé les CV obtenus avec 3 ou 2 jours par répétition,le nombre de répétitions par période passant ainsi de 3 à 4.

\section{RÉSULTATS ET DISCUSSION}

Les vaches ont ingéré $22,5 \pm 1,7 \mathrm{~kg}$ de matière sèche en $298 \pm 33 \mathrm{~min}$ et donné en moyenne $33229 \pm 7734$ coups de mâchoire pendant $549 \pm 98$ min de rumination. Ces durées journalières d'ingestion et de rumination sont semblables à celles obtenues par Dulphy et al (1990). Aucune relation significative $(P>0,05)$ n'a pu être établie entre la rumination, soit la durée ou la somme des coups de mâchoire pendant $24 \mathrm{~h}$, et l'ingestion volontaire, bien que ces paramètres varient $(P<0,05)$ d'une vache à l'autre.

\section{Validation}

Le coefficient de régression entre le compte fait visuellement pendant 3,6 ou 9 min de rumination (65 \pm 10 coups de mâchoires $\mathrm{min}^{-1}$ ) et le nombre d'interruptions enregistré par le capteur (69 \pm 12 coups de mâchoires min-1) $^{-1}$ n'a pas été différent de 1 $(P>0,05)$. L'erreur résiduelle représente un coefficient de variation de $2,9 \%$ et a été plus élevée pendant $3 \mathrm{~min}$ que pendant 9 min. L'algorithme a ignoré les CMR qui ont eu lieu pendant la dernière minute des variations du poids de l'auge causées par l'observateur. Cependant, en dehors de la période de validation, les variations de poids proviennent de périodes d'ingestion et ces périodes sont séparées par plus d'une minute des périodes de rumination. II est donc important que la vache ne soit pas en contact avec l'auge pendant la période de rumination.

Le coefficient de régression entre les sommes des ingestions de matière sèche $\min ^{-1}\left(22,5 \pm 1,7 \mathrm{~kg} \mathrm{j}^{-1}\right)$ et les quantités calculées en soustrayant les refus des quantités distribuées $\left(21,70 \pm 2,07 \mathrm{~kg} \mathrm{j}^{-1}\right)$ n'a pas été $(P>0,05)$ différent de 1 avec un coefficient de variation résiduel égal à $2,2 \%$.

Sur une base humide, l'erreur de pesée, c'est-à-dire la variation aléatoire de la lecture du poids, est de $150 \mathrm{~g}$. Pendant les périodes stables de pesée, le poids diminuait d'environ $100 \mathrm{~g}$ par $6 \mathrm{~h}$, probablement en raison de l'évaporation d'eau et de la dérive des balances. Cette dernière a été évaluée à $-20 \mathrm{~g} \mathrm{j}^{-1}$ en se basant sur le poids des auges vides.

\section{Variabilité du comportement alimentaire}

Les profils journaliers des activités d'ingestion (fig 3) et du mérycisme (fig 4) ont été représentés à l'aide des estimés obtenus par la méthode des moindres carrés de la procédure GLM de SAS (1987).

Avec 3 j par répétition, le coefficient de variation du profil d'ingestion et celui de la rumination, évalués à l'aide de l'interaction entre l'heure, la répétition et les vaches dans le dispositif en split-plot, ont été de 


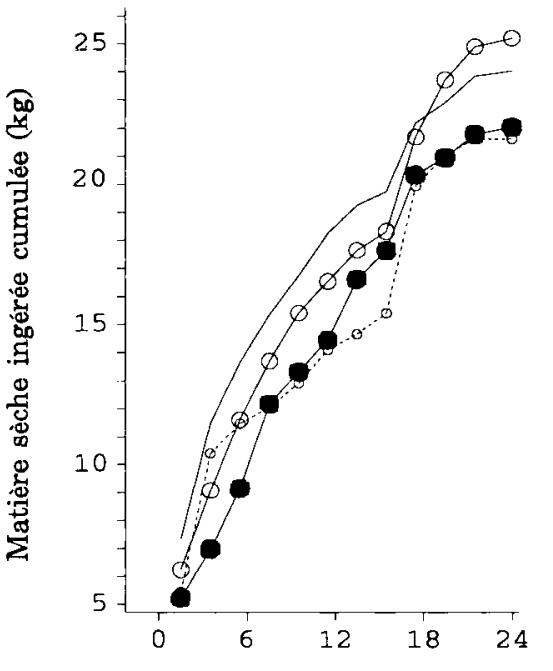

Heures depuis le premier repas

Fig 3. Évolution de la matière sèche ingérée cumulée au cours des $24 \mathrm{~h}$ qui suivent la distribution à $14 \mathrm{~h}$ d'une ration complète à base d'ensilage de fléole. - vache 5 ; - $-0--0$ vache $6 ;-0-0-$ vache $14 ; \longrightarrow-$ vache 22.

$4,1 \%$, et de $8,7 \%$ respectivement. Les répétitions de 2 j ont donné des résultats très semblables, soit $3,9 \%$ et $9,4 \%$. Cependant ce n'est qu'avec les répétitions de $3 \mathrm{j}$ que les profils d'ingestion ont varié avec les vaches $(P<0,05)$. Les profils de rumination ont toujours varié $(P<0,05)$ avec les vaches. Les profils de rumination ont été plus variables que ceux d'ingestion, mais l'effet des individus sur l'aspect des profils est plus important au niveau de la rumination qu'au niveau de l'ingestion.

\section{CONCLUSION}

La technique proposée mesure de manière précise la vitesse d'ingestion d'aliments et

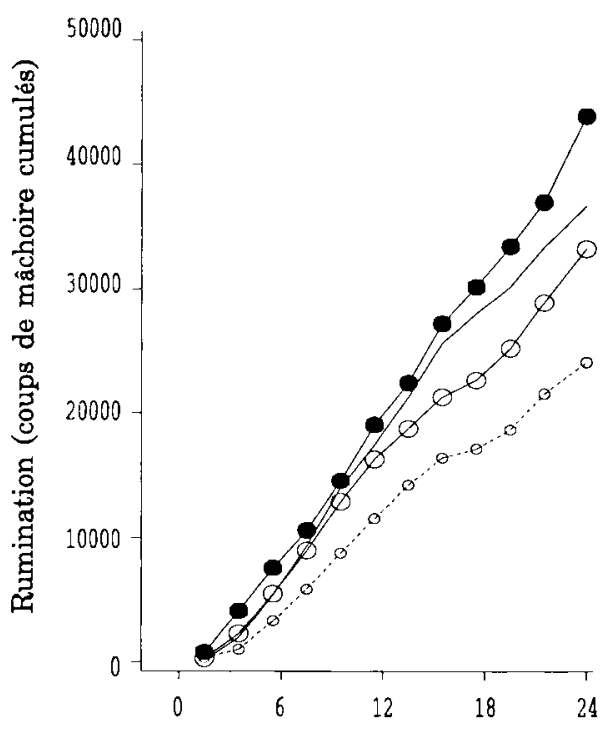

Heures depuis le premier repas

Fig 4. Évolution de la mastication pendant la rumination cumulée au cours des $24 \mathrm{~h}$ qui suivent la distribution à $14 \mathrm{~h}$ d'une ration complète à base d'ensilage de fléole.

vache $5 ;-0-10-$ vache $6 ;-0-0-$ vache $14 ; \longrightarrow-$ vache 22 .

l'activité mérycique. Les faibles coefficients de variations individuels nous permettent de conclure à la répétabilité des comportements alimentaires individuels pendant des périodes de $9 \mathrm{j}$. La répétabilité rend possible l'étude dans le temps des variations causées par une modification contrôlée des aliments. Cette méthodologie devrait permettre de mieux comprendre les facteurs qui limitent la capacité d'ingestion.

\section{RÉFÉRENCES}

Baumont R, Brun JP, Dulphy JP (1989) Influence of the nature of hay on its ingestibility and the kinetics of intake during large meals 
in sheep and cows. XVI International Grassland Congress, Nice

Beauchemin KA, Zelin S, Genner D, BuchananSmith JG (1989) An automatic system for quantification of eating and ruminating activities of dairy cattle housed in stalls. J Dairy Sci 72, 2746-2759

Dăberitz H, Prediger D, Dittrich A, Schirmbeck H, Hoffmann M, Winkelmann J (1989) Studies of the consumption and ruminating behaviour or sheep 5 . The continuous registration and recording of jaw movements of sheep caused by feed intake and ruminating on the basis of acceleration measurings. Arch Anim Nutr 6, 585-592

Deswysen AS (1987) Forestomach: control of digesta flow (LAA Ooms, AD Degryse, ASJPAM van Miert, eds) Martinius Nijhoff Publishers

Dulphy JP, Carle B, Demarquilly C (1990) A comparative study of feed intake and feeding activities in sheep, cattle and goats receiving stored forages with or without feed concentrate supplementation. 1. A descriptive study. Ann Zootech 39, 95-111

Hancock J (1953) Grazing behaviour of cattle. Anim Breed Abstr 21, 1

Luginbuh! JM, Pond KR, Russ JC, Bums JC (1987) A simple electronic device and computer interface system for monitoring chewing behavior of stall-fed ruminant animals. J Dairy Sci 70, 1307-1312

Martz FA, Belyea RL (1986) Role of particle size and forage quality in digestion and passage by cattle and sheep. J Dairy Sci 69, 19962008

Vasilatos R, Wangsness PJ (1980) Feeding behavior of lactating Dairy cows as measured by time-lapse photography. $J$ Dairy Sci 63 , $412-416$

SAS Institute Inc (1987) SAS/STAT Guide for personal computers, version 6 edition, SAS Institute Inc 